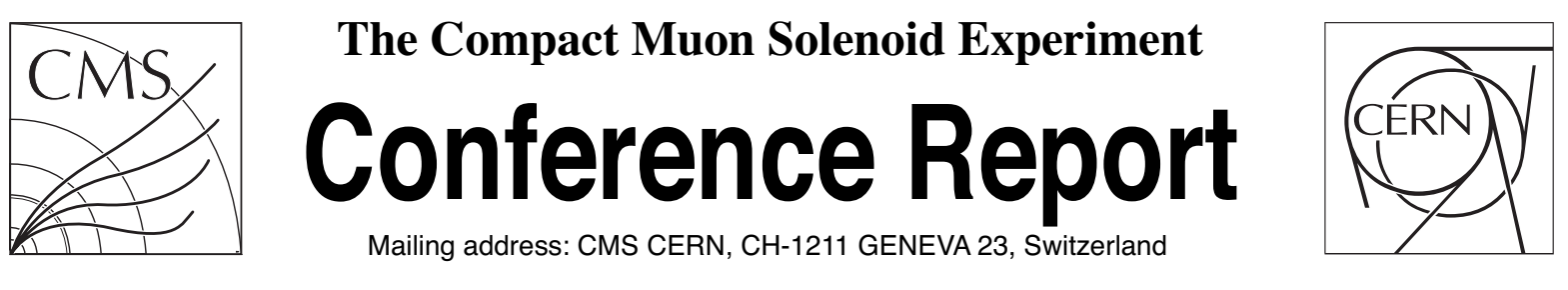

10 February 2020 (v4, 13 November 2020)

\title{
CMS ECAL upgrade for precision timing and energy measurements at the High-Luminosity $\mathrm{LHC}$
}

Riccardo Salvatico for the CMS Collaboration

\begin{abstract}
The High Luminosity upgrade of the LHC (HL-LHC) at CERN will provide unprecedented instantaneous and integrated luminosities of around $5 \times 10^{34} \mathrm{~cm}^{-2} \mathrm{~s}^{-1}$ and $3000 \mathrm{fb}^{-1}$, respectively, from 2025 to 2035. During this operational period, an average of 140 to 200 collisions per bunch-crossing (pile up) is expected, posing a challenge to the event reconstruction. In order to cope with these extreme pile up conditions, harsh environment, and increased data rates, the Compact Muon Solenoid (CMS) detector is undergoing a radical Phase II upgrade program. In the barrel region of the CMS electromagnetic calorimeter (ECAL), the lead tungstate crystals and avalanche photodiodes (APDs) will keep performing well and will therefore be maintained, while the entire readout and trigger electronics will be replaced. A dual gain trans-impedance amplifier and an ASIC providing two $160 \mathrm{MHz}$ ADC channels, gain selection, and data compression will be installed. The noise increase in the APDs, due to radiation-induced dark current, will be contained by reducing the temperature at which ECAL is operated. The trigger decision will be moved off-detector and performed by powerful and flexible FPGA processors, allowing for more sophisticated trigger algorithms to be applied.

The upgraded ECAL will be capable of high-precision energy measurements and will greatly improve the time resolution for photons and electrons above $10 \mathrm{GeV}$. Together with the introduction of a new timing detector designed to perform timing measurements with the resolution of a few tens of picoseconds for minimum ionizing particles, the CMS detector will be able to precisely reconstruct the primary interaction vertex under the described pile up conditions.
\end{abstract}

Presented at CHEF2019 Calorimetry for the High Energy Frontier 2019, Published in JINST 15 (2020) C05040, doi:10.1088/1748-0221/15/05/c05040 


\title{
CMS ECAL upgrade for precision timing and energy measurements at the High-Luminosity LHC
}

\author{
R. Salvatico on behalf of the CMS collaboration \\ Università degli Studi di Torino, \\ Via Giuria 1, Torino, Italy \\ INFN, \\ Via Giuria 1, Torino, Italy \\ E-mail: riccardo.salvatico@cern.ch
}

Abstract: The High Luminosity upgrade of the LHC (HL-LHC) at CERN will provide unprecedented instantaneous and integrated luminosities of around $5 \times 10^{34} \mathrm{~cm}^{-2} \mathrm{~s}^{-1}$ and $3000 \mathrm{fb}^{-1}$, respectively, from 2025 to 2035. During this operational period, an average of 140 to 200 collisions per bunch-crossing (pile up) is expected, posing a challenge to the event reconstruction. In order to cope with these extreme pile up conditions, harsh environment, and increased data rates, the Compact Muon Solenoid (CMS) detector is undergoing a radical Phase II upgrade program. In the barrel region of the CMS electromagnetic calorimeter (ECAL), the lead tungstate crystals and avalanche photodiodes (APDs) will keep performing well and will therefore be maintained, while the entire readout and trigger electronics will be replaced. A dual gain trans-impedance amplifier and an ASIC providing two $160 \mathrm{MHz}$ ADC channels, gain selection, and data compression will be installed. The noise increase in the APDs, due to radiation-induced dark current, will be contained by reducing the temperature at which ECAL is operated. The trigger decision will be moved offdetector and performed by powerful and flexible FPGA processors, allowing for more sophisticated trigger algorithms to be applied.

The upgraded ECAL will be capable of high-precision energy measurements and will greatly improve the time resolution for photons and electrons above $10 \mathrm{GeV}$. Together with the introduction of a new timing detector designed to perform timing measurements with the resolution of a few tens of picoseconds for minimum ionizing particles, the CMS detector will be able to precisely reconstruct the primary interaction vertex under the described pile up conditions.

Keywords: Calorimeters; Front-end electronics for detector readout 


\section{Contents}

1 The electromagnetic calorimeter of CMS at LHC 1

2 CMS at High-Luminosity LHC (HL-LHC) 1

2.1 ECAL at HL-LHC 2

3 ECAL electronics upgrade $\quad 2$

3.1 CATIA 3

3.2 LiTE-DTU 3

3.3 Front-End board 4

3.4 Off-detector electronics 4

4 Conclusions 6

\section{The electromagnetic calorimeter of CMS at LHC}

The ECAL [1] is a compact, hermetic, homogenous, high-granularity electromagnetic calorimeter, and it is part of the CMS experiment at LHC. It is composed of around 75 thousand lead tungstate $\left(\mathrm{PbWO}_{4}\right)$ crystals, distributed in a central barrel (EB), where they are read out by avalanche photodiodes (APDs), and in two endcaps (EEs), where they are read out by vacuum phototriodes (VPTs). The crystals are $2 \mathrm{~cm} \times 2 \mathrm{~cm} \times 23 \mathrm{~cm}$ in size and are characterized by a density of $8.28 \mathrm{~g} \mathrm{~cm}^{-3}$. Their short radiation length $X_{0}=0.89 \mathrm{~cm}$ allows the longitudinal shower containment within the $23 \mathrm{~cm}$ of crystal length, while the small Molière radius $r_{M}=2.19 \mathrm{~cm}$ ensures lateral shower containment and, therefore, high granularity.

ECAL has an excellent energy resolution, of a few percent for electrons above $\sim 45 \mathrm{GeV}$ and for photons above $\sim 60 \mathrm{GeV}$, and good time resolution, below 200 ps above $30 \mathrm{GeV}$. Such performances have contributed and still contribute to excellent physics results, from the SM precision measurements to searches for New Physics (NP).

\section{CMS at High-Luminosity LHC (HL-LHC)}

After 2026, the entire LHC physics program is going to be enriched with the upgrade of the accelerator to HL-LHC [2], a machine which will increase the instantaneous luminosity from $2 \times 10^{34}$ to $\sim 5 \times 10^{34} \mathrm{~cm}^{-2} \mathrm{~s}^{-1}$.

For CMS, this will imply the acquisition of an integrated luminosity $L_{\mathrm{int}} \simeq 3000 \mathrm{fb}^{-1}$ by the end of the data taking, allowing the measurement of Higgs couplings at the per cent level, the observation of $\mathrm{HH}$ production, searches for NP, etc. The detector will have to sustain extreme radiation levels, and to cope with a number of concurrent interactions per bunch crossing (pile up) increasing from an average of $40-60$ to an average of $140-200$. In such an extreme scenario, it will be fundamental to make the detector radiation-hard and to find new strategies to reconstruct the vertexes of interaction and to locate the primary vertex. 


\subsection{ECAL at HL-LHC}

Before the start of HL-LHC, the ECAL barrel will undergo a major upgrade [3], while the endcaps will be replaced by a new detector [4]. The readout electronics is being completely redesigned to obtain better time resolution and cope with the increase in latency (from $4.5 \mu$ s to $12.5 \mu \mathrm{s}$ ) and rate (from $100 \mathrm{kHz}$ to $750 \mathrm{kHz}$ ) of the CMS L1 trigger. The L1 trigger logic itself will be moved offdetector and evaluated by powerful and flexible FPGAs, allowing for more complex operations. The new electronics will be faster and it will implement a sampling frequency four folds increased with respect to the current one, that is from $40 \mathrm{MHz}$ to $160 \mathrm{MHz}$. These features will make it capable of a better time resolution, necessary to perform vertex location, in particular for photons, in a strongly pile-up-dominated environment. Another improvement they will bring is a better discrimination between scintillation light from electromagnetic showers in the calorimeter and direct hits in APDs, which cause abnormal signals (generally called spikes). The goal, supported by simulation studies, is a time resolution below $30 \mathrm{ps}$ for an energy deposition above $\sim 50 \mathrm{GeV}$.

It is expected that, by the end of the data taking with the HL-LHC, EB will have received a radiation dose up to $50 \mathrm{kGy}$. Even if such a dose is sustainable, the radiation-induced leakage current in the APDs would make the APD noise the dominant term in the energy resolution, in the current configuration. For this reason, the operation temperature of the barrel detector will be reduced from $18^{\circ} \mathrm{C}$ to $9^{\circ} \mathrm{C}$. Such change will also limit the losses in crystal light yield by $\sim 20 \%$.

The radiation damage affects the crystal transparency as well. In particular, the hadron-induced damage, differently from the electromagnetic damage, is not recoverable, and will be the dominant component at the HL-LHC. Nevertheless, it is estimated that the signal loss in the EB crystals (that is for a pseudorapidity $0 \leq|\eta| \leq 1.5$ ) will range from $55 \%$ to $70 \%$ at $\sim 3000 \mathrm{fb}^{-1}$ integrated luminosity; thus, the EB crystals will still perform fairly well and will not be replaced. On the other hand, the EEs will receive an unsustainable dose up to $10^{3} \mathrm{kGy}$. Therefore, they will be replaced by another detector, silicon and scintillator based.

\section{ECAL electronics upgrade}

In the next sections, an overview of the various components of the upgrade electronics will be given. A general, schematic representation is shown in figure 1.

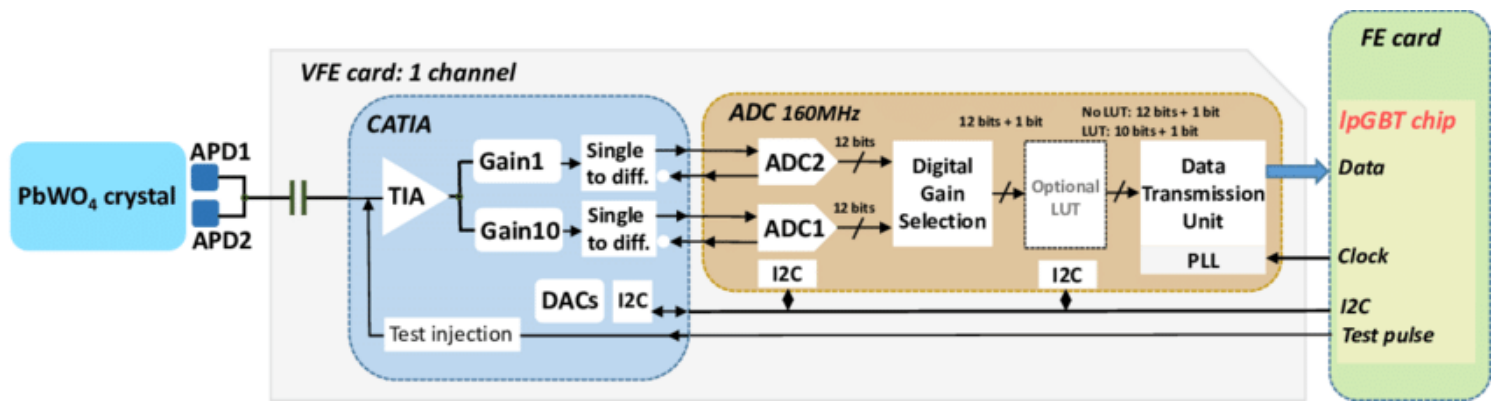

Figure 1. Overview of the ECAL upgrade electronics. 


\subsection{CATIA}

The first component of the upgraded VFE electronics is the CATIA, which stands for Calorimeter Trans-Impedance Amplifier. Based on a $130 \mathrm{~nm}$ CMOS technology, the CATIA is connected with the APDs through a Kapton wire and has $35 \mathrm{MHz}$ bandwidth. It implements two gains, 10 and 1, which are used to cover a dynamic range from $50 \mathrm{MeV}$ to $2 \mathrm{TeV}$. The board can perform test pulse injection for calibration.

The faster response of the CATIA with respect to the current amplifier allows a better discrimination between scintillation light and APD direct ionization.

Two versions of the CATIA boards have been tested in lab and during beam tests at CERN. The timing performance using crystals and APDs has been measured. The output signals were digitized at 5 GSample/sec using a CAEN V1742 module. Two micro-channel plates (MCP) were put in front of the crystal matrix to provide a reference time for each event. The constant term in the resolution curve was found to be about $20 \mathrm{ps}$, which makes it possible to reach the target of 30 ps resolution for electromagnetic energy depositions of several tens of $\mathrm{GeV}$.

Moreover, the tested boards showed excellent performance in terms of noise and linearity, and the energy resolution matched the one of the current electronics. Some of the results obtained during 2018 beam test are shown in figure 2 .
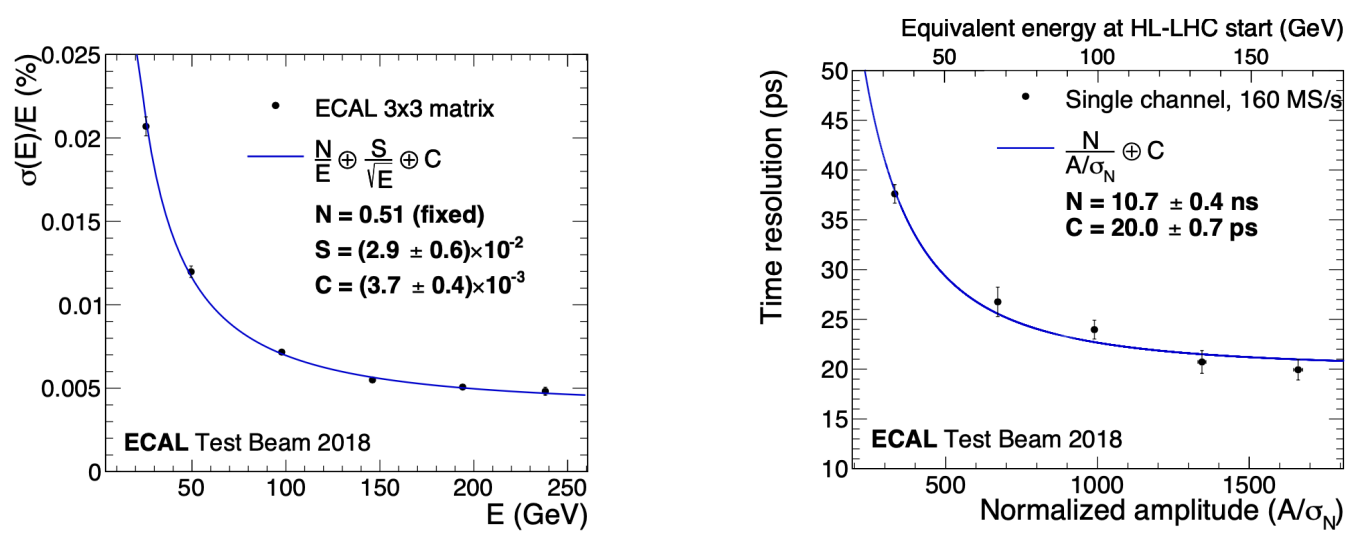

Figure 2. Energy and time resolution for the 2018 ECAL upgrade beam test setup.

\subsection{LiTE-DTU}

The analog signal processed by the CATIA is then elaborated by the LiTE-DTU, an acronym for Lisbon-Torino ECAL Data Transmission Unit. The LiTE-DTU is a data conversion and transmission ASIC, implemented in a $65 \mathrm{~nm}$ CMOS technology (see figure 3).

Its two-twelve bits ADCs receive the two analog signals from the preamplifier outputs (corresponding to the two gains) and convert them to a digital representation of the pulse, via voltage sampling. The sampling frequency is $160 \mathrm{MHz}$, in order to separate signals generated in the crystals from spikes. An internal clock running at $1.28 \mathrm{GHz}$ is used for serialization and is generated by a phase-locked loop (PLL) starting from the reference $160 \mathrm{MHz}$ clock. The data from the ADC connected to the high gain input are selected, unless the current sample or at least one sample in 


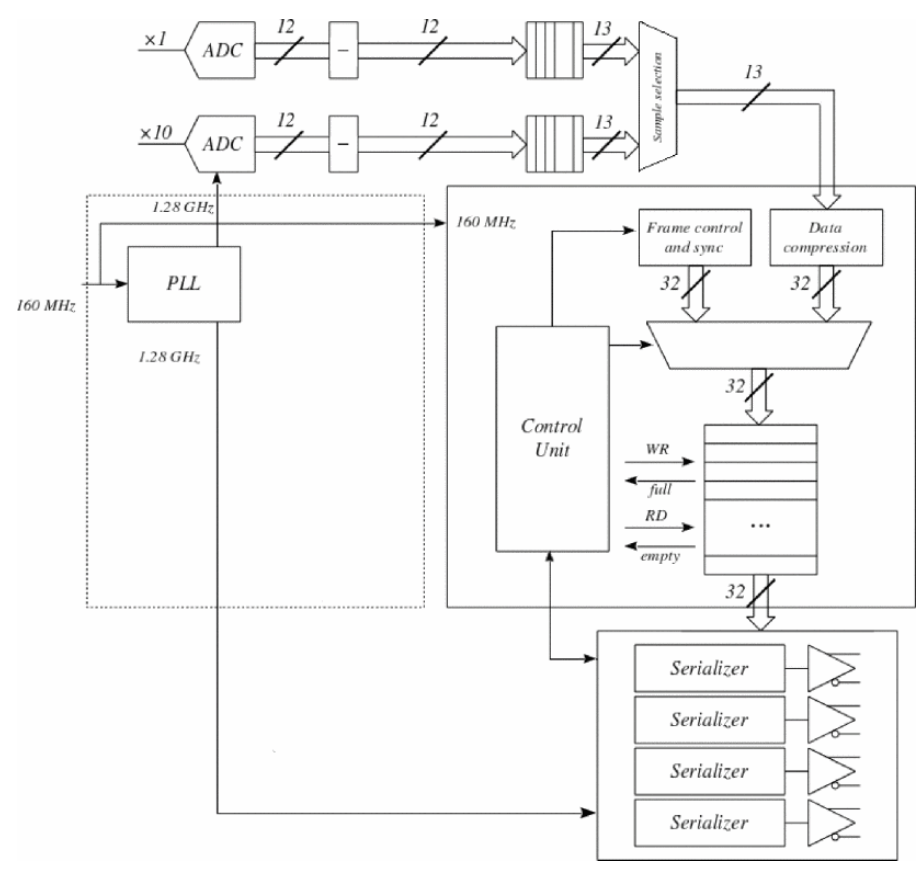

Figure 3. Schematics of the LiTE-DTU.

a certain time window around the current sample is saturated. This ensures that all the samples associated to the energy deposition of a particle are sent out with the same gain.

Data streams composed of $12(+1)$ bits (where the thirteenth bit indicates the gain value) are sent to the serializer, which is based on the e-link protocol of the lpGBT. While the e-link rate is $1.28 \mathrm{~Gb} / \mathrm{s}$, the transfer of the thirteen bit words at $160 \mathrm{MHz}$ generates a $2.08 \mathrm{~Gb} / \mathrm{s}$ occupancy. Therefore, a compression algorithm is adopted, based on a simplified Huffman encoding [5], which reduces the occupancy down to $1.08 \mathrm{~Gb} / \mathrm{s}$. The compression is possible since the energy spectrum of the hits in the calorimeter falls very rapidly, that is noise or low energy signals occupy most of the transmission bandwidth. Thus, the algorithm uses 6 bits to encode signals up to an energy of $2.5 \mathrm{GeV}$ energy, and $12(+1)$ bits for more energetic signals.

The first version of the LiTE-DTU is currently under test, and all the aforementioned benchmarks are being assessed, as well as the dose tolerance (expected to be up to $100 \mathrm{kGy}$ ) and the incidence of single event upsets (SEUs).

\subsection{Front-End board}

The Front-End is responsible of initializing and controlling all VFE components through an I2C interface. The FE precisely distributes the clock to all VFE cards and streams the digitized data to the Back-End (BE) electronics. Four uplinks are available for data streaming $(10.24 \mathrm{~Gb} / \mathrm{s})$ and one downlink for electronics control $(2.56 \mathrm{~Gb} / \mathrm{s})$.

\subsection{Off-detector electronics}

The CMS operations at HL-LHC will be characterized by a high $\mathrm{L} 1$ trigger rate of $750 \mathrm{kHz}$, and an increased trigger latency of $12.5 \mu \mathrm{s}$. Moreover, all the ECAL samples will be streamed off- 
detector, in order to perform complex operations such as spike rejection and pile-up subtraction online. Among the other advantages, this will allow to produce L1 trigger primitives at crystal level, whereas currently they are produced for a $5 \times 5$ crystal matrix.

In contrast with the current design, the upgraded off-detector electronics is conceived to sustain the higher L1 trigger rate, as well as to provide the required trigger latency and to shift the trigger primitive generation off-detector. A processor board called Barrel Calorimeter Processor (BCP) which implements these features is being designed with commercially-available FPGAs and high speed optical links. The BCP, designed for integration in a standard ATCA [6] crate, needs a powerful FPGA to analyze and decompress the received data, and transmit to the L1 trigger a pre-processed set of trigger primitives. At the same time, it must provide the clock and control to the FE board, and also interface with the DAQ. A schematic view of the FE-BCP-L1 chain is presented in figure 4.

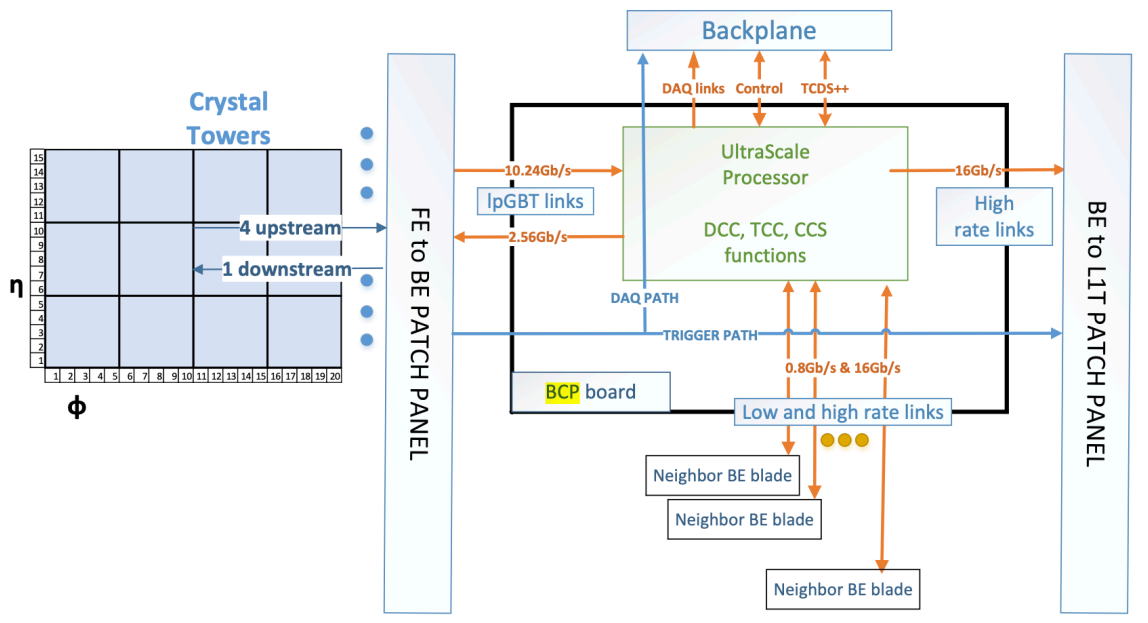

Figure 4. Schematic view of the FE-BCP-L1 chain. The trigger concentrator function (TCC) generates the trigger primitives for the $\mathrm{L} 1$ trigger. A data concentrator block (DCC) provides the L1 trigger latency data buffers and the status of the buffers. The clock and control system (CCS) distributes clock and control signals to the FE electronics.

The algorithms required to be implemented in this board include:

- rejection of signal induced by direct APD ionization (spikes);

- decompression and conversion of the digitized pulse data into transverse energy;

- basic clustering of localised energy;

- formation of the trigger primitives;

- generation of clock and control signals to be transmitted to the FE. 


\section{Conclusions}

The upgrade of the CMS ECAL foreseen for High-Luminosity LHC was presented. The upgrade will involve the central barrel of the calorimeter only, since the endcaps will not be able to sustain the harsh radiation environment, and will therefore be replaced by another calorimeter. The EB upgrade involves a complete redesign of the readout electronics, from the APD up, and the lowering of the operation temperature to half of the current value.

\section{References}

[1] CMS collaboration, The CMS electromagnetic calorimeter project: technical design report, CERN-LHCC-97-033, CERN, Geneva, Switzerland, 15 December 1997 [CMS-TDR-4].

[2] G. Apollinari et al., CERN yellow reports: monographs, volume 4 (2017): High-Luminosity Large Hadron Collider (HL-LHC) technical design report v.0.1, CYRM-2017-004, CERN, Geneva, Switzerland (2017).

[3] CMS collaboration, The phase-2 upgrade of the CMS barrel calorimeters, CERN-LHCC-2017-011, CERN, Geneva, Switzerland, 12 September 2017 [CMS-TDR-015].

[4] CMS collaboration, The phase-2 upgrade of the CMS endcap calorimeter, CERN-LHCC-2017-023, CERN, Geneva, Switzerland, 9 April 2018 [CMS-TDR-019].

[5] D. Huffman, A method for the construction of minimum-redundancy codes, Proc. IRE 40 (1952) 1098.

[6] PICMG, Reference. PICMG 3.0 revision 2.0 AdvancedTCA base specification, http://www.picmg.org. 